Please do not remove this page

RMIT

UNIVERSITY

\title{
A simple auxetic tubular structure with tuneable mechanical properties
}

Ren, Xin; Shen, Jianhu; Ghaedizadeh, Arash; Tian, Hongqi; Xie, Yi Min

https://researchrepository.rmit.edu.au/esploro/outputs/9921860212501341/filesAndLinks?institution=61RMIT_INST\&index=null

Ren, X., Shen, J., Ghaedizadeh, A., Tian, H., \& Xie, Y. M. (2016). A simple auxetic tubular structure with tuneable mechanical properties. Smart Materials and Structures, 25(6), 1-9.

https://doi.org/10.1088/0964-1726/25/6/065012

Document Version: Accepted Manuscript

Published Version: https://doi.org/10.1088/0964-1726/25/6/065012

Repository homepage: https://researchrepository.rmit.edu.au

(C) 2016 IOP Publishing Ltd

Downloaded On 2023/04/26 19:46:17 +1000

Please do not remove this page 
Thank you for downloading this document from the RMIT Research Repository.

The RMIT Research Repository is an open access database showcasing the research outputs of RMIT University researchers.

RMIT Research Repository: http://researchbank.rmit.edu.au/

\begin{abstract}
Citation:
Ren, X, Shen, J, Ghaedizadeh, A, Tian, H and Xie, Y 2016, 'A simple auxetic tubular structure with tuneable mechanical properties', Smart Materials and Structures, vol. 25 , no. 6, 065012, pp. 1-9.
\end{abstract}

See this record in the RMIT Research Repository at:

https://researchbank.rmit.edu.au/view/rmit:37184

Version: Accepted Manuscript

Copyright Statement:

(C) 2016 IOP Publishing Ltd

Link to Published Version:

http://dx.doi.org/10.1088/0964-1726/25/6/065012 


\title{
A simple auxetic tubular structure with tuneable mechanical properties
}

\author{
Xin Ren ${ }^{1,2}$, Jianhu Shen ${ }^{1}$, Arash Ghaedizadeh ${ }^{1}$,Hongqi Tian ${ }^{2}$, and Yi Min Xie ${ }^{1,3 *}$ \\ ${ }^{1}$ Centre for Innovative Structures and Materials, School of Engineering, RMIT University, GPO Box 2476, Melbourne 3001, \\ Australia \\ ${ }^{2}$ Key Laboratory of Traffic Safety on Track, School of Traffic \& Transportation Engineering, Central South University, \\ Changsha 410075, Hunan Province, China \\ ${ }^{3}$ XIE Archi-Structure Design (Shanghai) Co., Ltd., 1436 Jungong Road, Yangpu District, Shanghai 200433, China
}

\begin{abstract}
:
Auxetic materials and structures are increasingly used in various fields because of their unusual properties. Auxetic tubular structures have been fabricated and studied due to their potential to be adopted as oesophageal stents where only tensile auxetic performance is required. However, studies on compressive mechanical properties of auxetic tubular structures are limited in the current literature. In this paper, we developed a simple tubular structure which exhibits auxetic behaviour in both compression and tension. This was achieved by extending a design concept recently proposed by the authors for generating 3D metallic auxetic metamaterials. Both compressive and tensile mechanical properties of the auxetic tubular structure were investigated. It was found that the methodology for generating 3D auxetic metamaterials could be effectively used to create auxetic tubular structures as well. By properly adjusting certain parameters, the mechanical properties of the designed auxetic tubular structure could be easily tuned.
\end{abstract}

Keywords: Auxetic, Tubular structure, Metallic metamaterial, Plastic deformation, Buckling

\section{Highlights:}

1) A simple auxetic tubular structure which exhibits auxetic behaviour in both compression and tension has been designed and fabricated.

2) The latest methodology for generating auxetic metallic metamaterials has been adopted and successfully extended to tubular structures.

3) Both tensile and compressive mechanical properties for the simple axuxetic tubular structure have been investigated.

4) Key parameters for tuning the mechanical properties of metallic tubular structures have been identified and examined. 


\section{Introduction}

Materials and structures with negative Poisson's ratio (NPR) exhibit counter-intuitive behaviour, i.e. under uniaxial compression (tension), these materials and structures contract (expand) transversely. They are also named as "auxetics" by Evans [1].

Because of the uncommon feature which is equipped by auxetics, these auxetic materials and structures are superior to conventional materials and structures in terms of indentation resistance [2, 3], shear resistance [4], synclastic behaviour [5], enhanced resilience [5], energy absorption [6-9], fracture toughness [10] and vibration control [11].

As one branch of auxetics, auxetic tubular structure or auxetic stent has attracted much research effort towards exploring its applications as foldable devices in the medical field, such as angioplasty stents [12-14], annuloplasty rings [15] and oesophageal stents [16, 17]. For the patterns of all these medical devices, the cellular configuration is predesigned. The conventional straight forward method is to roll the 2D auxetic sheets to tubes. However the cellular tubes designed in this method may not have auxetic behaviour under large compressive strain. Besides, most of the existing auxetic stents only demonstrate auxetic behaviour in tension. To the best knowledge of authors, no systematic research has been carried out on auxetic tubular structures under large compressive strain which is required when stents scaffold are inserted to the blood vessels. Recently, Mohsenizadeh et al [8] concluded that auxetic foam-filled square tube is superior to empty and conventional foamfilled square tubes in terms of crashworthiness indicators through experimental method, and Hou et al [9] obtained the optimal Poisson's ratio of the filled material for three foamfilled tubes in terms of energy absorption though FE method. However, all the tubes they used were conventional one, investigation on auxetic tubes are rare, particularly in compression.

Inspired by a planar auxetic metamaterial induced by elastic instability [18-20], we selected an available void fraction of 0.69 (larger than 0.34 in [18]), which demonstrated bucklinginduced auxetic behaviour, to generate a tubular structure with normal circular holes. During FE simulations, we found that when the base material of the designed tubular structure is rubber, the tubular structure did show auxetic behaviour, as expected. However, this auxetic behaviour disappeared when the base material was replaced with brass.

In this paper we implement the latest methodology of generating buckling-induced auxetic metamaterials proposed in our previous paper[21], to generate a simple auxetic tubular structure which could be easily tuned by one parameter named as the pattern scale factor (PSF). An in-depth investigation on the designed auxetic tubular structure is carried out both experimentally and numerically. Although the focus point is on the compressive auxetic properties of the designed structure, tensile auxetic performance is also investigated. A series of parametric studies on the designed auxetic tubular structure are executed by using the experimentally validated FE models. 


\section{Designing auxetic tubular structures}

Similar to our previous work [21], the methodology of generating auxetic tubular structures can be summarized as four steps. Firstly, designing buckling-induced auxetic tubular structure; secondly, carrying on buckling analysis of the initial tubular structure with linear elastic base material; thirdly, identifying the desirable buckling mode; lastly, altering the initial tubular structure using the desirable buckling mode.

\subsection{Designing buckling-induced auxetic tubular structure}

The first step of the design framework is to generate a buckling-induced auxetic tubular structure. Similar to the geometry configuration of the Bertoldi's work [18], a planar sheet is shown in figure 1(a) with a void fraction of 0.69. Under planar constraint, it demonstrates a negative Poisson's ratio behaviour induced by elastic instability. Using coordinate transformation method, the planar sheet can be transferred into a tubular structure as shown in figure $1(\mathrm{~b})$.

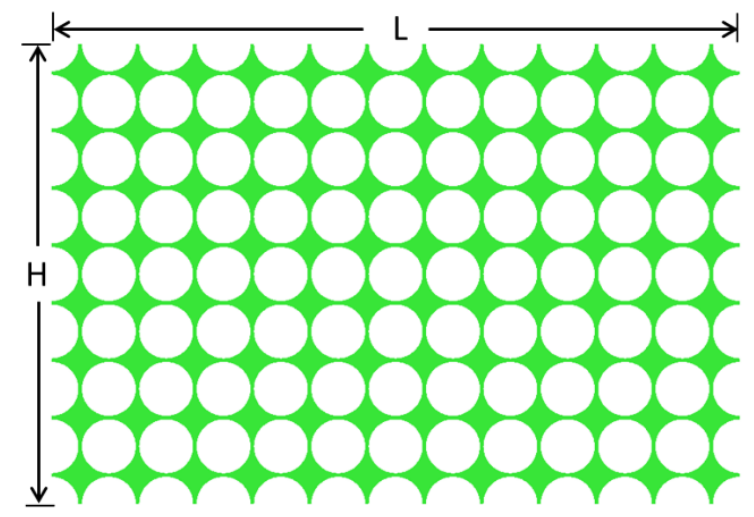

(a)

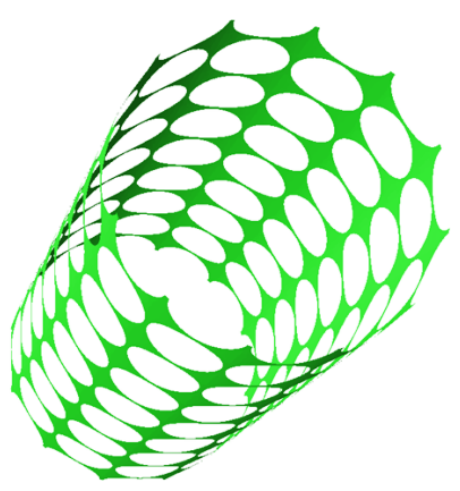

(b)

Figure 1. Geometries of planar sheet with auxetic behaviour induced by elastic instability ( $L=147 \mathrm{~mm}, H=98$ $\mathrm{mm}$, void fraction=0.69): (a) the planar sheet with periodic circular holes; (b) the corresponding tubular structure.

\subsection{Buckling analysis of the initial tubular structure with linear elastic base material}

The second step is to perform buckling analysis to obtain desirable buckling modes with auxetic performance under uniaxial compression. The modulus of $110 \mathrm{GPa}$ and the Poisson's ratio of 0.38 were used in the simulation. Lanczos was chosen as the eigensolver in buckling analysis. In this work, the out-of-plane rotational degree of freedom on top and bottom nodes of the FE model was constrained. The degree of freedom of bottom nodes along compressive direction was also constrained. The nodal movement on the top nodes was allowed to move in the loading direction. It should be noted that one of nodes in the bottom was fixed to avoid rigid rotation. Shell elements were used for buckling analysis.

The commercial finite element software package ABAQUS (Simulia, Providence, RI) was implemented for running buckling analysis. ABAQUS/Standard was adopted for linear 
buckling analysis using a Lanczos eigensolver. The number of eigenvalues requested was set as 10. The tubular structure was built using shell elements (ABAQUS element type SA) with shell thickness of $6 \mathrm{~mm}$. Symmetrical mesh seed was distributed to the FE model which sustained uniaxial compressive force.

\subsection{Identifying the desirable buckling mode}

The desirable buckling mode was selected based on similar patterns observed from previous research on planar auxetic structures. To be more specific, the configuration of the anticipated buckling modes should contain geometry similar to the alternating ellipsoidal pattern in our previous and other's work [18, 20-27]. The results of the first two buckling modes are presented in figure 2 . Apparently, the first buckling mode meets the requirement of selecting desirable modes, while the second buckling mode is unqualified because the holes are irregular.

Mode 1

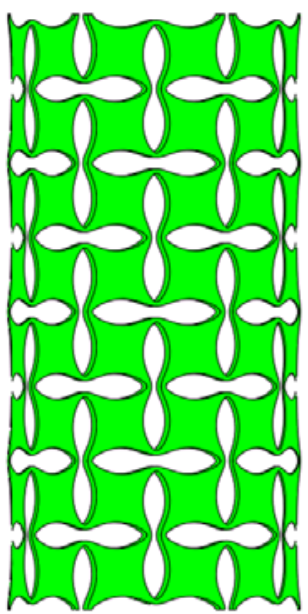

(a)
Mode 2

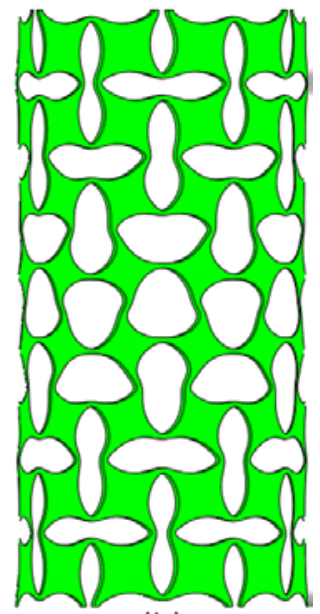

(b)

Figure 2. The first two buckling modes of the initial tubular structure: (a) configuration of the first bucklingmode; (b) configuration of the second buckling mode.

\subsection{Quantifying the desirable buckling mode and form the auxetic tubular structure}

The method of quantifying the desirable buckling mode is similar to our previous work [21]. The pattern scale factor (PSF) was employed to quantify the adjustment on the initial tubular structure using the desirable buckling mode. In the present work, when the edge of the elliptical void is just closed, as shown in figure 3, the corresponding deformation scale factor (DSF) ( 0.01465 in this case) is defined as PSF $=100 \%$. Other values of PSF are defined accordingly, such as $50 \%$ with a deformation scale factor of 0.007325 , and the $0 \%$ is the initial tubular structure without any adjustment.

Because the result of the first buckling mode obtained from buckling analysis was uniform. Unlike our previous work [21] where a representative volume element (RVE) was employed, 
the adjustment was directly applied into the whole configuration of the initial tubular structure in this study.
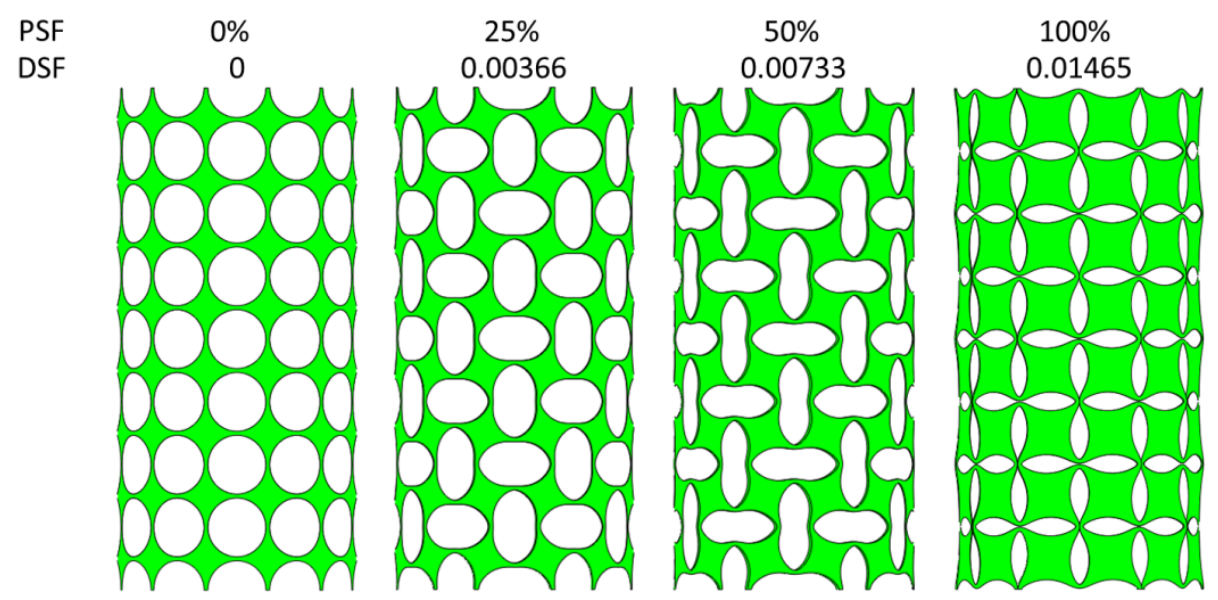

Figure 3. The geometries of the first buckling mode at different values of PSF from $0 \%$ to $100 \%$.

\section{Experiment}

\subsection{Fabrication of metallic tubular structure for experiments}

The specimens of the tubular structure in figure 4 were fabricated using 3D printing (Shapeways, New York) technique with raw brass as their base material. The specific manufacturing procedure of the metallic tubular structure was same as our previous work [21]. The material properties of the printed raw brass material were measured through standard tensile tests which was completed in the previous work [21].

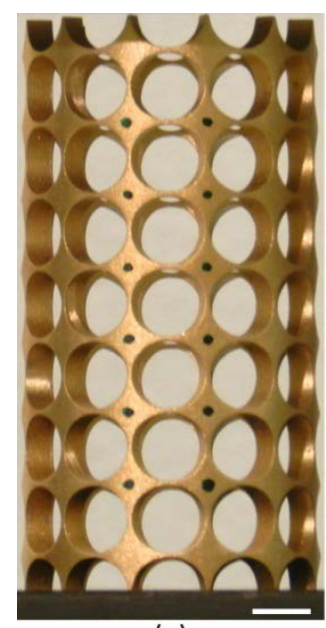

(a)

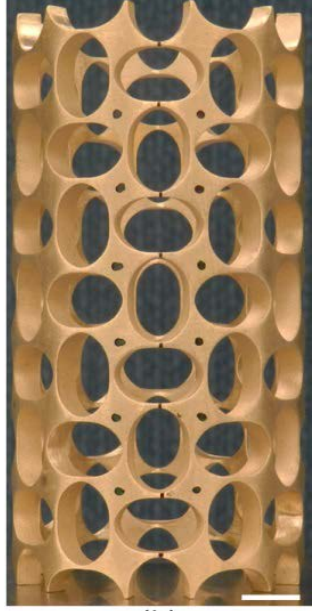

(b)

Figure 4. Two test samples for the tubular structure (scale bar: $10 \mathrm{~mm}$ ): (a) initial tubular structure $(P S F=0 \%)$; (b) altered tubular structure (PSF=20\%). Unixial compression tests on tubular structures

The mechanical performance of the printed tubular structures was tested using standard quasistatic uniaxial compression tests, and the strain rate of $10^{-3} \mathrm{~s}^{-1}$ was employed using a Shimazu machine. A camera was used to record the deformation procedure to measure the evolution of the Poisson's ratio of the tubular structures. 
As can be seen in figure 4, the centres of two rotation part of six layers were marked with small points. The experimental value of Poisson's ratio was calculated using image processing method from 6 layers of the tubular structures, as shown in figure 5. The equation of calculating Poisson's ratio of one layer of the tubular structure is shown in formula (1) and the equation of calculating overall Poisson's ratio of the tubular structure is shown in formula (2).

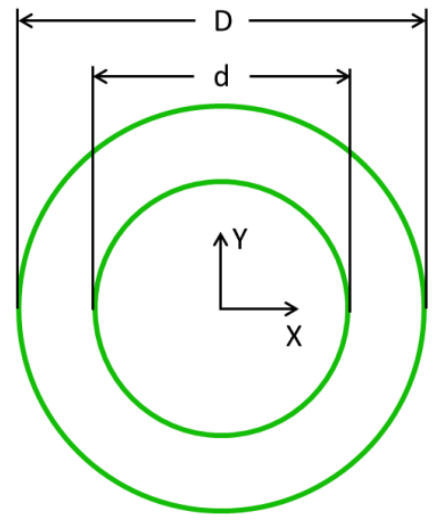

(a)

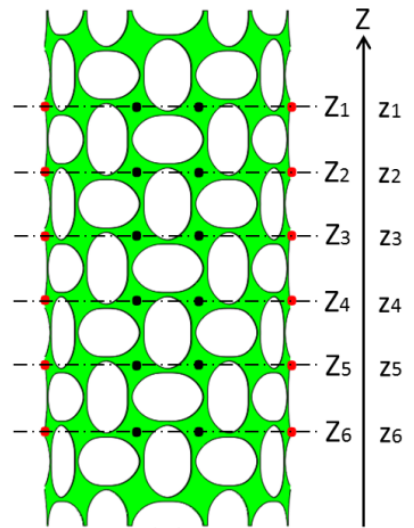

(b)

Figure 5. Calculation Poisson's ratio using an imaging processing method from two perspective views: (a) top view; (b) front view.

$$
\begin{array}{ll}
v_{i}=-\frac{\varepsilon_{x y}}{\varepsilon_{z}}=-\frac{\Delta d / D}{\Delta z_{i} / z_{i}} & (2 \leq \mathrm{i} \leq 5) \\
\bar{v}=\frac{1}{4} \times \sum_{2}^{5} v_{i} & (2 \leq \mathrm{i} \leq 5)
\end{array}
$$

Where $\Delta d=d-D, Z_{i}=Z_{i-1}-Z_{i+1}, \Delta z=z_{i-1}-z_{i+1}$

\subsection{The comparison on auxetic behaviour of the two tubular structures from experiments}

According to the Bertoldi's finding in terms of negative Poisson's ratio behaviour induced by elastic instability [18], here we utilized a similar geometry mentioned in their work to generate a tubular structure as shown in figure 4(a). In our previous study [21], we found that the loss of auxetic behaviour in metallic auxetic metamaterials. To verify the loss of auxetic behaviour will also occur towards tubular structures, here we made a comparison experimentally.

The experimental deformation processes for two tubular metallic samples of $\mathrm{PSF}=0 \%$ and $\mathrm{PSF}=20 \%$ are illustrated in figure 6 . The initial metallic tubular structure with $\mathrm{PSF}=0 \%$ is non-auxetic, and the altered metallic tubular structure with $\mathrm{PSF}=20 \%$ illustrates auxetic behaviour. This result further verified that the phenomenon of the loss of auxetic behaviour not only occurs for 3D metamaterials, but also exists for tubular structures. In addition, using the latest methodology of generating 3D metallic metamaterials, the loss of auxetic behaviour towards tubular structure could be easily obtained again. 


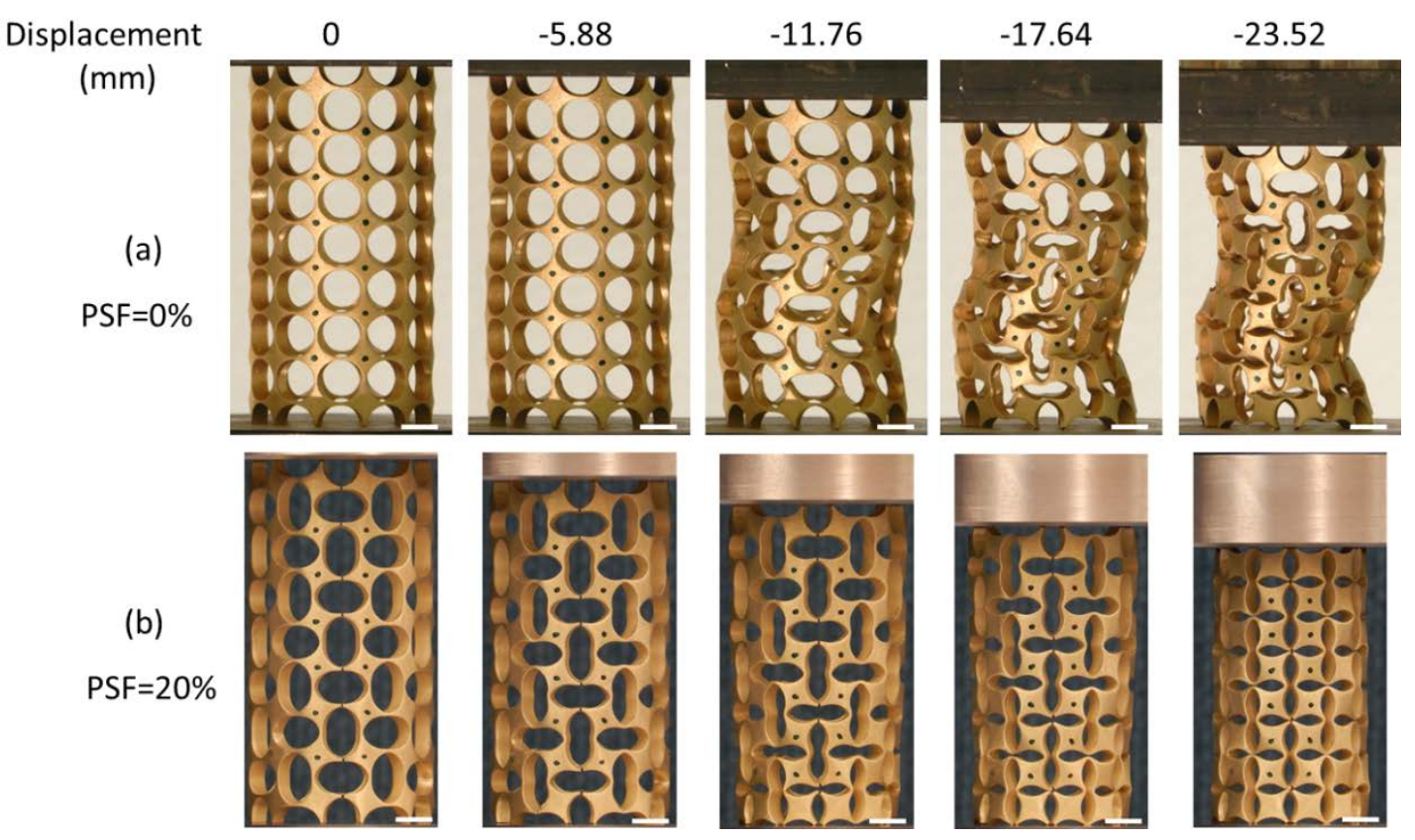

Figure 6. Experiments on two metallic tubular structures (scale bar: $10 \mathrm{~mm}$ ): (a) global buckling of the metallic sample with $\mathrm{PSF}=0 \%$; (b) auxetic behaviour of the metallic sample with $\mathrm{PSF}=20 \%$.

According to the method of calculating Poisson's ratio described in figure 5 and formulas (1)-(2), the value of Poisson's ratio for the tubular structure with $\mathrm{PSF}=0 \%$ could not be calculated properly because the marked points in the same layer were not in same horizontal level. The ideal auxetic deformation pattern is that the diameter of the tubular structures in different height could change evenly under uniaxial deformation. But from the deformation pattern shown in 6(a), we can define the metallic tubular structure with $\mathrm{PSF}=0 \%$ is non-auxetic. The experimental values of Poisson's ratio as a function of displacement are shown in figure 7.

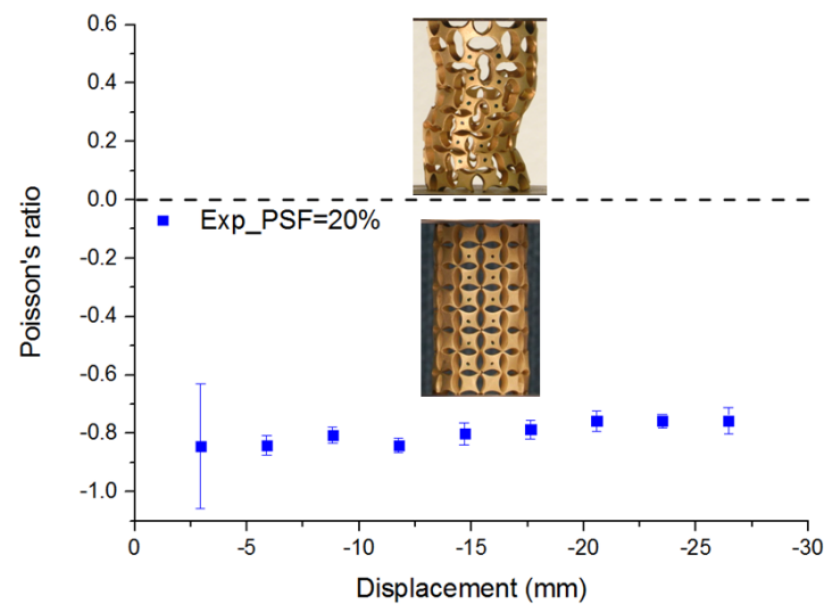

Figure 7. Experimental results of Poisson's ratio as a function of displacement for the model with PSF=20\%.

So far, the experimental result has confirmed that the methodology of generating 3D metallic metamaterials can be extended to design auxetic tubular structure as well. To the best knowledge of authors, no one has conducted studies on auxetic tubular structures about their compressive mechanical performance. To illustrate the nonlinear effect on metallic auxetic tubular structures, numerical investigation both on compressive and tensile auxetic performance was executed on our 
designed tubular structures. The influence of PSF and metal plasticity on their mechanical properties was explored.

\section{Finite element analysis}

\subsection{FE model for metallic tubular structure}

The geometric configuration of the FE model is shown in figure 5. ABAQUS/Explicit solver was employed in post-buckling analysis for considering large deformation and self-contacts [28]. Although the shell elements were used in the stage of generating auxetic tubular structures, for obtaining more accurate result in FE simulations, solid elements (ABAQUS element type C3D8) were adopted for large deformation analysis. Because the base material of the experimental specimens were same as that of the printed models in our previous work [21], the same bilinear elastic-plastic material model with a Young's modulus of $87 \mathrm{GPa}$ and a hardening modulus of $1.7 \mathrm{GPa}$ were used in FE simulations.

Through comparing the FE models and the printed tubular models, we found that the printed tubular models were lighter than our initial design. The relative mass errors were $9.0 \%$ and $12.3 \%$ for the two printed samples with PSF of $0 \%$ and $20 \%$, respectively. We found that the wall thickness of the printed models were smaller than our initial design. Adjustment of the FE models was made by decreasing the thickness of the tubular structures when it is compared with experimental results.

\subsection{FE model validation}

The mesh dependence analysis was performed, which is similar to the work conducted by Pozniak et al [29]. A mesh size with four layers of elements for the minimal link of the FE models was adopted. The FE model was validated by comparing the deformation process and force-displacement curves from the simulation with that from experiments. The deformation process of the tubular structure with $\mathrm{PSF}=20 \%$ was shown in figure 8 , which is nearly identical to the deformation process from experiments shown in figure $6(\mathrm{~b})$.

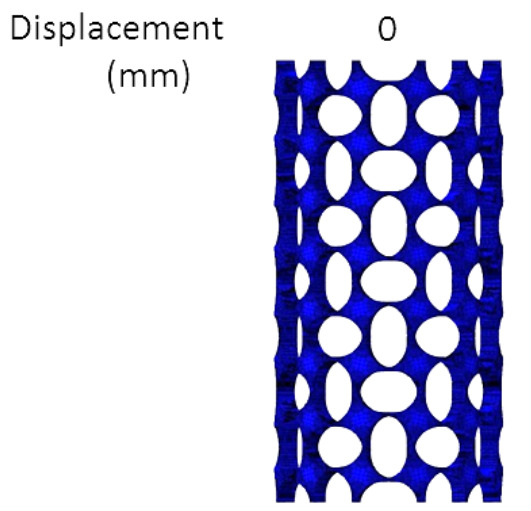

$-5.88$

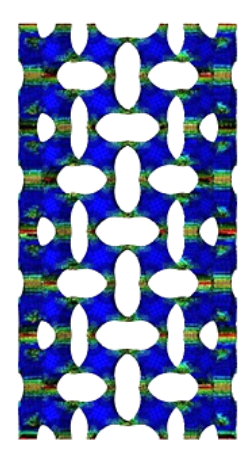

$-11.76$

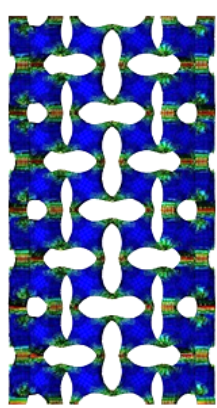

$-17.64$

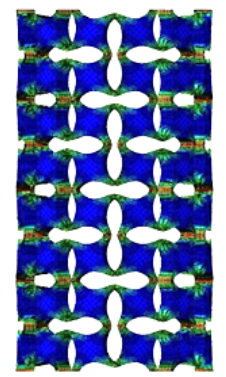

$-23.52$

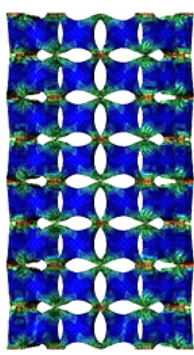

Figure 8. Deformation process of the FE model with $\mathrm{PSF}=20 \%$.

The FE model was further validated by comparing the force-displacement curves as shown in figure 9 . Although the peak force of the experimental result is higher than that of FE result, the overall trends of these two curves are similar. Through checking the geometries of the printed sample after test, we found some broken parts on it. However, the failure criterion was not defined in the FE 
simulations. Therefore, we attributed the difference between these two curves to the imperfection of the printed specimen and the fracture of the minimal links in experiment, which are difficult to simulate using FE models.

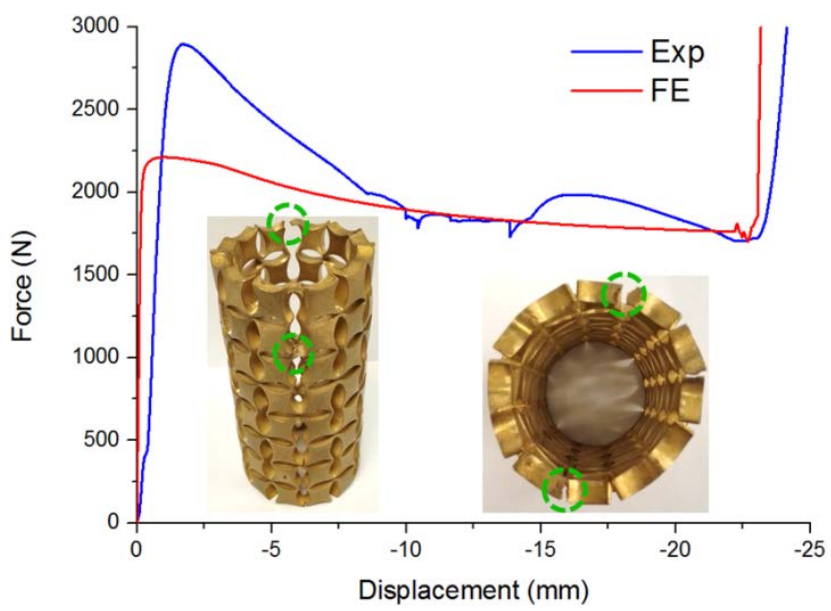

Figure 9. Comparison of the force-displacement curves of the tubular structure with $\mathrm{PSF}=20 \%$, between experiment and FE simulation. (The images of the printed sample after the experiment are shown, where the green dashed circles illustrate the broken parts.)

\subsection{Comparison of rubber and brass tubular structures}

According to the previous findings of our work [21], the initial auxetic behaviour of the bucklinginduced metamaterial disappeared when the base material of rubber was replaced by brass. Based on the work of Bertoldi et al [18], we selected the similar geometry with a void fraction of 0.69 , to generate a tubular structure which should possess the auxetic behaviour induced by elastic instability. To verify the effect of base material on the tubular structure with $\mathrm{PSF}=0 \%$, we conducted a comparison using FE method by changing material model in ABAQUS setting. The linear elastic material model for rubber with a Yong's modulus of $1 \mathrm{MPa}$ was chosen in FE simulations. The deformation patterns for the same geometry with two different material models were shown in figure 10.

The FE results illustrate that the base material has a significant effect on the auxetic performance of the buckling-induced tubular structure. When the base material is replaced from rubber to brass, the initial auxetic behaviour of buckling-induced tubular structure would disappear. The finding is similar to the finding we have observed for the test of 3D auxetic metamaterials. 


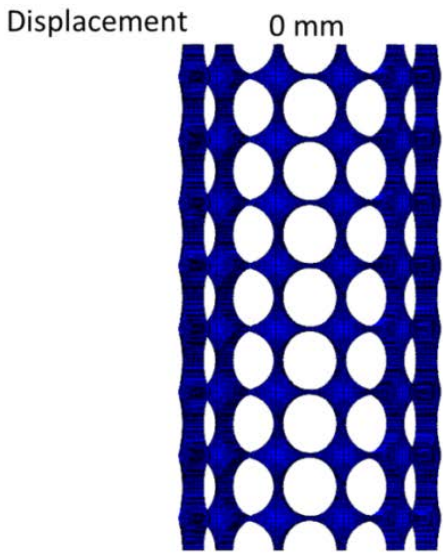

(a)
$-7.3 \mathrm{~mm}$

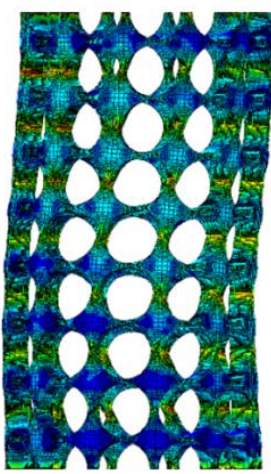

(b)
$-7.3 \mathrm{~mm}$

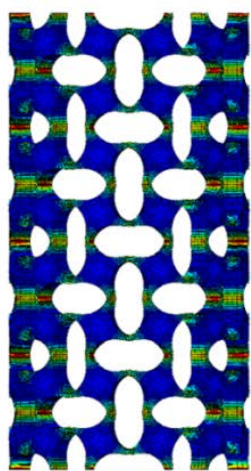

(c)

Figure 10. Illustration of different deformation patterns of the tubular structure with PSF= $0 \%$ with different base materials" (a) undeformed shape;(b) FE model using a linear elastic material model for rubber; (c) FE model using elasto-plastic material model with isotropic linear strain hardening, for brass.

\subsection{Effect of PSF on auxetic behaviour}

The magnitude of the PSF not only determines the geometric configuration of the designed tubular structure but also affects auxetic performance of the metallic tubular structure. The auxetic performance for different models with various values of PSF was investigated using the validated FE models.

The variation of the auxetic performance with respect to the values of PSF is shown in figure 11. We can see clearly that the auxetic performance of the tubular structure can be adjusted through changing the values of PSF. By increasing the value of PSF, the effective strain range for the tubular structure under tension could be enlarged, while the effective strain range under compression will become smaller. Therefore, in order to obtain an axetic tubular structure which has a similar effective auxetic strain both in compression and tension, a proper value of PSF should be chosen. As can be seen in the figure $11(\mathrm{a})$ and 11 (b), when the tubular structure has a PSF of $60 \%$, the trends of the curves of Poisson's ratio-displacement and force-displacement are nearly the same.

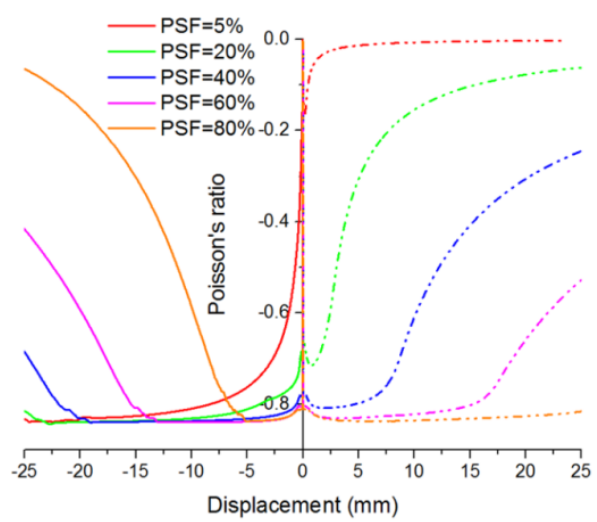

(a)

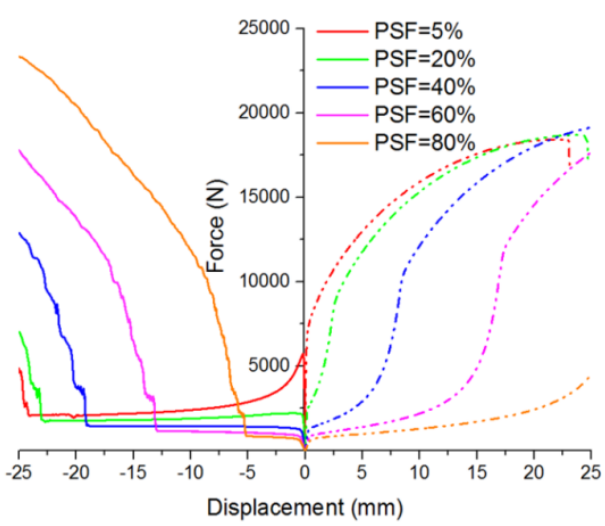

(b)

Figure 11. Effect of PSF on mechanical performances both in compression and tension: (a) curves of Poisson's ratio as a function of displacement for metallic tubular structures with different PSF, (b) curves of force as a function of displacement for metallic tubular structures with different PSF. 


\subsection{Effect of plastic strain hardening on auxetic performance}

Because of the high ductility of raw brass (with an elongation up to 0.3 ), it was chosen as the base material of the printed tubular structures. Strain hardening, as a fundamental feature of metal plasticity, has a significant effect on the mechanical performance of cellular structures and materials. The effect of plastic strain hardening on auxetic performance and load-bearing capability of the designed tubular structures was investigated by using the validated FE models. A bilinear elasticplastic material model was employed in FE models. The FE results of the parametric study for strain hardening are shown in figure 12, where Es is the elastic modulus and Ep is the strain hardening modulus.

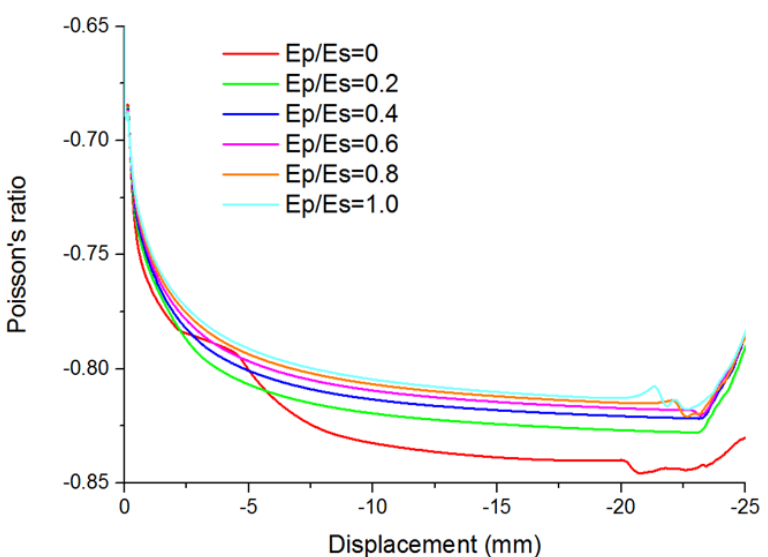

(a)

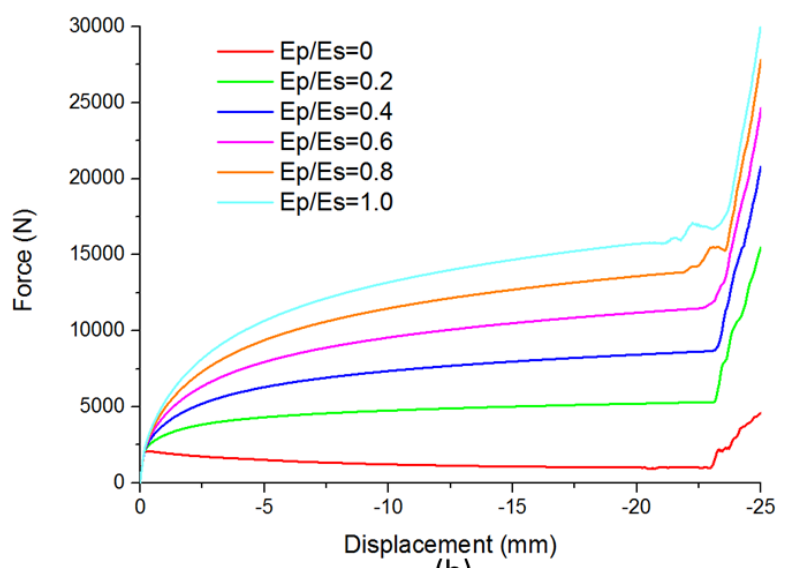

(b)

Figure 12. Effect of plastic strain hardening on mechanical properties of designed tubular structures: (a) Poisson's ratio as a function of displacement for models with PSF $=20 \%$ and base material with different values of Ep/Es; (b) force as a function of displacement for models with $\mathrm{PSF}=20 \%$ and base material with different values of Ep/Es.

\section{Concluding remarks}

In this study, the latest methodology for generating 3D auxetic metamaterials was successfully extended to the development of metallic auxetic tubular structures. The performance of a simple metallic auxetic tubular structure under both compressive and tensile loading was investigated experimentally and numerically. The effects of the pattern scale factor (PSF) and the plastic strain hardening on the auxetic performance and other mechanical properties were examined using the validated FE models. From the obtained results, the following conclusions could be drawn:

(a) A simple auxetic tubular structure has been designed, fabricated and tested, which exhibits auxetic behaviour in both compression and tension.

(b) The buckling-induced auxetic tubular structure would lose its auxetic behaviour when the base material is changed from an elastomer to a ductile metal.

(c) The latest methodology for generating 3D metallic auxetic metamaterials has been further developed to create a simple auxetic tubular structure, and the effectiveness of the design approach has been validated experimentally and numerically.

(d) The mechanical properties of the proposed tubular structure can be tuned by adjusting the magnitude of the PSF. When the PSF is set at a certain value ( $60 \%$ in this study), it is possible to achieve a similar auxetic performance under compression and tension. 
The most significant feature of our designed auxetic tubular structure is its tunability by simple control parameters, i.e. the PSF and the plastic strain hardening ratio of base material. The mechanical properties of the tubular structure could be easily adjusted by these two parameters individually. Most of the existing auxetic stents only exhibit auxetic behaviour under tension. The designed simple tubular structure has auxetic behaviour in both compression and tension. Therefore, our designed metallic tubular structure not only has potential to be used in the medical field, but could also be employed in other structures (e.g. in armoured vehicles to absorb impact loading). The same design approach could be extended to the development of new composite materials and structures with auxetic behaviour.

\section{Acknowledgments}

This work was supported by the Australian Research Council (DP140100213), the China Scholarship Council (201306370057), the Major Program of the National Natural Science Foundation of China (U1334208).

\section{References}

[1] K.E. Evans, M.A. Nkansah, I.J. Hutchinson, S.C. Rogers, Nature, 353 (1991) 124-124.

[2] I.I. Argatov, R. Guinovart-Díaz, F.J. Sabina, International Journal of Engineering Science, 54 (2012) 42-57.

[3] V.L. Coenen, K.L. Alderson, Physica Status Solidi B, 248 (2011) 66-72.

[4] J.B. Choi, R.S. Lakes, Journal of Materials Science, 27 (1992) 4678-4684.

[5] R.S. Lakes, Science, 235 (1987) 1038-1040.

[6] K.E. Evans, Endeavour, 15 (1991) 170-174.

[7] A. Alderson, K.L. Alderson, Proceedings of the Institution of Mechanical Engineers, Part G: Journal of Aerospace Engineering, 221 (2007) 565-575.

[8] S. Mohsenizadeh, R. Alipour, M. Shokri Rad, A. Farokhi Nejad, Z. Ahmad, Materials \& Design, 88 (2015) 258-268.

[9] S. Hou, T. Liu, Z. Zhang, X. Han, Q. Li, Materials \& Design, 82 (2015) 247-259.

[10] J.B. Choi, R.S. Lakes, International Journal of Fracture, 80 (1996) 73-83.

[11] C.P. Chen, R.S. Lakes, Journal of Engineering Materials and Technology, 118 (1996) 285-288.

[12] K. Kuribayashi, K. Tsuchiya, Z. You, D. Tomus, M. Umemoto, T. Ito, M. Sasaki, Materials Science and Engineering: A, 419 (2006) 131-137.

[13] K. Kuribayashi, Z. You, Google Patents, 2006.

[14] T.J. Ley, G.L. Kveen, T.G.J. Ehr, B.J. Brown, D.L. Friesen, Google Patents, 2002.

[15] G. Burriesci, G. Bergamasco, Google Patents, 2011.

[16] M.N. Ali, I.U. Rehman, Journal of Materials Science: Materials in Medicine, 22 (2011) 2573-2581.

[17] M. Ali, J.C. Busfield, I. Rehman, Journal of Materials Science: Materials in Medicine, 25 (2014) 527-553.

[18] K. Bertoldi, P.M. Reis, S. Willshaw, T. Mullin, Advanced Materials, 22 (2010) 361-366.

[19] K. Bertoldi, M.C. Boyce, S. Deschanel, S.M. Prange, T. Mullin, Journal of the Mechanics and Physics of Solids, 56 (2008) 2642-2668.

[20] T. Mullin, S. Willshaw, F. Box, Soft Matter, 9 (2013) 4951-4955.

[21] X. Ren, S. Jianhu, G. Arash, T. Hongqi, Y.M. Xie, Smart Materials and Structures, 24 (2015) 095016.

[22] M. Taylor, L. Francesconi, M. Gerendás, A. Shanian, C. Carson, K. Bertoldi, Advanced Materials, 26 (2014) 2365-2370.

[23] J.T.B. Overvelde, K. Bertoldi, Journal of the Mechanics and Physics of Solids, 64 (2014) 351-366.

[24] J.T.B. Overvelde, S. Shan, K. Bertoldi, Advanced Materials, 24 (2012) 2337-2342. 
[25] P. Wang, J. Shim, K. Bertoldi, Physical Review B, 88 (2013) 014304.

[26] S. Willshaw, T. Mullin, Soft Matter, 8 (2012) 1747-1750.

[27] J. Shen, S. Zhou, X. Huang, Y.M. Xie, Physica Status Solidi B, 251 (2014) 1515-1522.

[28] A.G. Hanssen, O.S. Hopperstad, M. Langseth, H. Ilstad, International Journal of Mechanical Sciences, 44 (2002) 359-406.

[29] A.A. Pozniak, K.W. Wojciechowski, Physica Status Solidi B, 251 (2014) 367-374. 